Classification

Physics Abstracts

$34.50 \mathrm{~L}$

\title{
Ion-quadrupole effects in thermal energy ion-molecule collision rate constants
}

\author{
A. M. Kosmas \\ Department of Chemistry, University of Ioannina, Greece
}

(Reçu le 11 avril 1985, accepté sous forme définitive le 8 juillet 1985)

\begin{abstract}
Résumé. - Une version simplifiée de la théorie de l'Orientation Quadrupolaire Moyenne (AQO) est développée en considérant la méthode de l'invariant adiabatique, pour étudier l'effet du potentiel d'interaction d'un ion avec une molécule quadrupolaire sur la constante de vitesse de la réaction en énergie thermique. La théorie est exprimée au moyen d'une moyenne de l'énergie potentielle et elle prédit une augmentation considérable du taux de capture au-dessus de la valeur de Langevin pour des molécules ayant des grands moments quadrupolaires et des petites polarisabilités. Les résultats sont comparés à plusieurs données expérimentales et aux prévisions d'autres théories.
\end{abstract}

\begin{abstract}
A simplified version of the Averaged Quadrupole Orientation(AQO) theory is attempted, by taking into account the adiabatic invariance approximation, to investigate the effect of the ionquadrupolar molecule interaction on thermal energy reaction rate constant. The theory is formulated in terms of an orientation averaged potential energy function and predicts a significant increase in the capture rate constant over the Langevin value for molecules with large quadrupole moments and small polarizabilities. The results of the theory are compared to various experimental data and predictions of other theories.
\end{abstract}

\section{Introduction.}

Several classical collision theories have been developed in recent years to calculate the increase in the magnitude of the capture rate constant over the Langevin value due to ion-dipolar molecule effects. Less attention has been devoted to ion-molecule collisions where the molecule possesses only a quadrupole moment. The ion-quadrupolar molecule interaction is usually weaker than the ion-dipole one and results in a smaller effect on the thermal reaction rate. Nevertheless this effect may become appreciable when the neutral molecule has a large quadrupole moment and a small polarizability. For molecules with a charge distribution cylindrically symmetric about the $z$ axis, which are the type of interest here this interaction is given by

$$
V_{Q}(r, \vartheta)=\left(q Q / 4 r^{3}\right)\left(3 \cos ^{2} \vartheta-1\right)
$$

where $q$ is the charge on the ion, $r$ is the distance between the ion and the centre of mass of the molecule, $\vartheta$ is the angle between the $z$ axis and the internuclear distance and $Q$ is the static qua- 
drupole moment of the molecule. Sometimes equation (1) is expressed in terms of the rotation averaged quadrupole moment having about half the value of the static one [1] as

$$
V_{Q}(r, \vartheta)=\left(q Q / 2 r^{3}\right)\left(3 \cos ^{2} \vartheta-1\right) .
$$

Dugan and Palmer [2] investigated very early the ion-quadrupole effects via numerical calculations of capture cross sections and computer-plotter studies of ion trajectories in the quadrupole field. A significant treatment that deals with ion-quadrupole capture collisions is the AQO theory of Su and Bowers [3, 4]. The AQO model is based on the same assumptions and approximations as the ADO theory for ion-dipole collisions and predicts the thermal rate coefficient fairly accurately. Recently a statistical approach was developed based on a thermodynamic treatment of ion-molecule interactions with good results [5]. Also Takayanagi [6] used the perturbed rotational state (PRS) method to treat low velocity collisions between ions and nonpolar molecules with large quadrupole moments. He calculated hitting cross sections for ion- $\mathrm{CO}_{2}$ capture collisions using the adiabatic potential $\varepsilon_{j m}(r)$ in the Langevin formulation.

In the theory of Su and Bowers the angle $\vartheta$ that the molecular axis makes with the line of centres of the collision is treated in the " average sense "; thus the orientation factor of the potential is substituted by a function of $r$ resulting by averaging $\vartheta$ over all possible orientations allowed by energy criteria. A critical assumption of the model is that conservation of energy allows to express the instantaneous rotational energy in the plane normal to the line of centres as

$$
E(r, \vartheta)=E(\infty)-V(r, \vartheta)
$$

where $E(\infty)$ is the initial rotational energy of the neutral molecule, i.e., the value of $E(r, \vartheta)$ at infinite separation. In an important paper referring to ADO theory [7] Bates has shown that this assumption is not valid since the averaged orientation factor does not take the proper limiting value for $E(\infty)$ going to zero. Instead he introduced the " adiabatic invariant approximation " which gave excellent results [8]. Later his work was generalized by Sakimoto [9] who incorporated it into the semiclassical perturbed-rotational state theory (PRS) to obtain the rate coefficient of orbiting collisions between ions and polar molecules.

In the present paper an improvement is attempted of the AQO theory based on the new developments in ion-molecule collision theory. Following Bates we employ the adiabatic invariant approximation to calculate the average orientation factor $\left\langle\cos ^{2} \vartheta\right\rangle$ rather $\operatorname{than}\langle\vartheta\rangle$ as in AQO theory.

The resulting average ion-quadrupole interaction is a function of the initial rotational energy of the molecule. By integrating this potential over the Boltzmann distribution of rotational energies, a function of $r$ only results which is then used in the Langevin theory to obtain the rate coefficient.

\section{Formulation.}

From equation (1) we see that the ion-quadrupole interaction ranges from the minimum value $-q Q / 4 r^{3}$ for $\vartheta=\pi / 2$ up to the maximum value $q Q / 2 r^{3}$ for $\vartheta=0$. When the molecule possesses no initial rotational energy it lines itself at an orientation $\vartheta=\pi / 2$ along the minimum of the potential energy function. As soon as $E(\infty)$ rises the molecule starts to librate around the position of minimum energy between $\vartheta^{*}$ and $\pi-\vartheta^{*}$ which correspond to the maximum value of $\cos ^{2} \vartheta$ allowed by energy criteria. When the initial rotational energy exceeds the barrier of the ionquadrupole interaction, the molecule has enough energy to swing around a full rotation over $2 \pi$. The dominant contribution to the rate coefficient comes from the region

$$
q Q / 2 r^{3} \geqslant E(\infty)
$$


in which the rotor librates keeping

$$
\cos ^{2} \vartheta \leqq \cos ^{2} \vartheta^{*}
$$

We may express the instantaneous rotational kinetic energy in terms of the rotational energy along the minimum of the ion-quadrupole interaction, $E(r, \pi / 2)$, which yields for $\cos ^{2} \vartheta^{*}$

$$
\cos ^{2} \vartheta^{*}=4 r^{3} E(r, \pi / 2) / 3 q Q \text {. }
$$

The average orientation factor $\left\langle\cos ^{2} \vartheta\right\rangle$ may then be calculated by means of equation (6). From the physical consideration of the problem $\left\langle\cos ^{2} \vartheta\right\rangle$ must tend to zero as $E(\infty)$ decreases.

Bates and Sakimoto pointed out that if $p$ be the orbital angular momentum and $q$ be the conjugate coordinate then

$$
J=\oint p \mathrm{~d} q / 2 \pi
$$

is an adiabatic invariant of the motion. This allows the rotational energy for a given $E(\infty)$ to be computed, by expressing the invariant in terms of the complete elliptic integrals of the first and second kind [10]. The computational procedure is quite involved and in view of the smaller effect of the ion-quadrupole interaction on the rate coefficient some approximations may be used. When applying (7) we may confine our attention to the simple case in which the axis of the rotating molecule is perpendicular to the orbital plane. Equation (7) takes the form

$$
J=\int_{\vartheta^{*}}^{\pi-\vartheta^{*}}\left[E(r, \pi / 2)-\frac{3 q Q}{4 r^{3}} \cos ^{2} \vartheta\right]^{1 / 2} \mathrm{~d} \vartheta / \pi
$$

where $\vartheta^{*}$ and $\pi-\vartheta^{*}$ are the turning points of the $\vartheta$ motion. When $r$ is infinite equation (8) readily yields

$$
J=[E(\infty)]^{1 / 2}
$$

An estimation of (8) for $r$ finite may be made by expressing $\cos ^{2} \vartheta$ in terms of the angle $\beta=$ $\pi / 2-\vartheta$. Since the libration is restricted mainly about $\vartheta=\pi / 2$, angle $\beta$ can be considered small which allows to put $\sin ^{2} \beta \approx \beta^{2}$. Thus from equation (8) we find approximately

$$
J \approx 2 E(r, \pi / 2) / 3^{1 / 2}\left(q Q / r^{3}\right)^{1 / 2}
$$

so that

$$
E(r, \pi / 2) \approx[E(\infty)]^{1 / 2} 3^{1 / 2}\left(q Q / r^{3}\right)^{1 / 2} / 2
$$

From the AQO theory the average orientation factor is expressed as

$$
\left\langle\cos ^{2} \vartheta\right\rangle=\int P(\vartheta) \cos ^{2} \vartheta \mathrm{d} \vartheta / \int P(\vartheta) \mathrm{d} \vartheta
$$

where

$$
\begin{aligned}
P(\vartheta) & \propto \sin \vartheta / \dot{\vartheta} \\
& \propto \sin \vartheta /(E(r, \vartheta))^{1 / 2}
\end{aligned}
$$

or in terms of $E(r, \pi / 2)$

$$
P(\vartheta) \propto \sin \vartheta /\left[E(r, \pi / 2)-\frac{3 q Q}{4 r^{3}} \cos ^{2} \vartheta\right]^{1 / 2},
$$


$\vartheta$ being the angular velocity of the $\vartheta$ motion. Substituting into equation (12) and performing the integrations yields

$$
\left\langle\cos ^{2} \vartheta\right\rangle=E(r, \pi / 2) /\left(\frac{3}{2} \frac{q Q}{r^{3}}\right)
$$

which in view of equation (11) shows that $\left\langle\cos ^{2} \vartheta\right\rangle$ is zero for $E(\infty)$ going to zero. Substituting equation (16) into equation (1) results in an $r$-dependent average ion-quadrupole interaction $V_{Q}(r)$ given by

$$
V_{Q}(r)=E(r, \pi / 2) / 2-q Q / 4 r^{3} .
$$

Combining equation (11) and performing the integration over the Boltzmann distribution of the initial rotational energies for the allowed range of energies finally yields

$$
V_{Q}(r, T)=-0.023 \frac{q^{2} Q^{2}}{r^{6} k T} .
$$

It is interesting to note at this point that equation (18) for the thermal average of the ionquadrupole interaction agrees well with the average free energy function obtained by Celli $e t$ al. using the thermodynamic approach. In their work they define a partition function for a system with temperature dependent energy levels as

$$
f(r, T)=\frac{\int_{0}^{\pi} \mathrm{e}^{-\psi(r, \vartheta) / k T} \sin \vartheta \mathrm{d} \vartheta}{\int_{0}^{\pi} \sin \vartheta \mathrm{d} \vartheta}
$$

where in the present case $\psi(r, \vartheta)$ stands for the ion-quadrupole interaction energy. The average free energy function

yields

$$
F(r, T)=-k T \ln f(r, T)
$$

$$
F_{Q}(r, T) \approx-q^{2} Q^{2} / 40 r^{6} k T
$$

which is used in the activated complex theory to derive the rate constant. Thus our results are expected to be close to those derived by the thermodynamic approach.

Equation (18) is next used in the Langevin formulation. The effective potential becomes

$$
U(r)=\frac{E b^{2}}{r^{2}}-\frac{\alpha q^{2}}{2 r^{4}}-0.023 \frac{q^{2} Q^{2}}{r^{6} k T}
$$

where $E$ is the initial kinetic energy of the system and $\alpha$ the polarizability of the neutral. The condition for orbiting collisions is that at the separation $r_{0}$ corresponding to the top of the centrifugal barrier two equations must be satisfied :

and

$$
\mathrm{d} U / \mathrm{d} r=0
$$

$$
U\left(r_{0}\right)=E \text {. }
$$


After the cross section $\sigma(v)$ is determined the rate constant is calculated from

$$
k(T)=\int_{0}^{\infty} v \sigma(v) P(v) \mathrm{d} v
$$

where $v$ is the initial relative velocity and $P(v)$ is the Boltzmann distribution of initial velocities. Solution of equations (23) and (24) by iteration procedure yields the cross section which from equation (25) gives the thermal rate constant as

$$
k(T)=k_{\mathrm{L}}\left(1+0.0146 \frac{Q^{2}}{(k T)^{1 / 2} q \alpha^{3 / 2}}\right)
$$

where $k_{\mathrm{L}}$ is the Langevin value for the rate coefficient.

\section{Results.}

Tables I and II compare results of the present theory to the experimental data and the predictions of other theories. We see that the agreement is generally good. Although the rate constants calculated by the present treatment do not differ much from the AQO results, we believe that it is an improvement to the AQO theory because it is based on more realistic assumptions than the AQO model. It must be mentioned that the AQO method distinguishes two cases in the use of the ionquadrupole potential function depending upon the sign of the ion charge and the sign of the quadrupole moment. When they are different the AQO model predicts lower effect on the reaction rate than in the $q Q>0$ case and thus its validity is questionable [11]. We think that the thermally

Table I. - Rate constant ratios for exothermic proton transfer reactions of $\mathrm{C}_{2} \mathrm{H}_{2}\left({ }^{a}\right)$ with various ions, at $300 \mathrm{~K}$.

\begin{tabular}{l|cccc}
\multicolumn{1}{c|}{ Ion } & Expt/Langevin $\left({ }^{b}\right)$ & Expt/Stat $\left({ }^{c}\right)$ & Expt/AQO $\left(^{d}\right)$ & Expt/Present \\
\hline $\mathrm{H}_{3}^{+}$ & 1.11 & 0.91 & 0.96 & 0.99 \\
$\mathrm{D}_{3}^{+}$ & 1.20 & 0.98 & 1.03 & 1.06 \\
$\mathrm{CH}_{5}^{+}$ & 1.17 & 0.96 & 1.01 & 1.04 \\
$\mathrm{~N}_{2} \mathrm{H}^{+}$ & 1.27 & 1.00 & 1.05 & 1.08 \\
$\mathrm{HCO}^{+}$ & 1.18 & 0.96 & 1.01 & 1.05 \\
$\mathrm{CO}_{2} \mathrm{H}^{+}$ & 1.30 & 1.06 & 1.12 & 1.16 \\
$\mathrm{~N}_{2} \mathrm{OH}^{+}$ & 1.15 & 0.94 & 0.99 & 1.02 \\
$\mathrm{H}^{-}$ & 1.01 & 0.83 & 0.97 & 0.90 \\
$\mathrm{D}^{-}$ & 1.06 & 0.87 & 1.00 & 0.94 \\
$\mathrm{NH}_{2}^{-}$ & 1.35 & 1.10 & 1.29 & 1.20 \\
$\mathrm{CH}_{3} \mathrm{NH}^{-}$ & 1.15 & 0.95 & 1.11 & 1.02 \\
$\mathrm{C}_{2} \mathrm{H}_{5} \mathrm{NH}^{-}$ & 1.11 & 0.91 & 1.06 & 0.99 \\
$\left(\mathrm{CH}_{3}\right)_{2} \mathrm{~N}^{-}$ & 0.98 & 0.80 & 0.94 & 0.87 \\
$\mathrm{OH}^{-}$ & 1.64 & 1.34 & 1.56 & 1.45 \\
$\mathrm{CH}_{3} \mathrm{O}^{-}$ & 1.44 & 1.18 & 1.38 & 1.27 \\
$\mathrm{C}_{2} \mathrm{H}_{5} \mathrm{O}^{-}$ & 1.34 & 1.09 & 1.27 & 1.19
\end{tabular}

( $\left.{ }^{a}\right)$ Static quadrupole moment of $\mathrm{C}_{2} \mathrm{H}_{2}, 7.2 \times 10^{-26}$ e.s.u. $\mathrm{cm}^{2}$.

(b) Experimental data from Ref. [11].

(c) Results from Ref. [5].

$\left({ }^{d}\right)$ Results from Ref. [11]. 
Table II. - Rate constant ratios for exothermic proton transfer reactions of $D_{3}^{+}$with neutral molecules at $300 \mathrm{~K}$.

\begin{tabular}{l|ccc}
\multicolumn{1}{c|}{ Neutral } & Expt/Langevin $\left(^{b}\right)$ & Expt/AQO $\left(^{b}\right)$ & Expt/Present \\
\hline $\mathrm{C}_{2} \mathrm{H}_{2}(7.2)\left({ }^{a}\right)$ & 1.24 & 1.06 & 1.10 \\
$\mathrm{C}_{2} \mathrm{~N}_{2}(-9.0)$ & 1.06 & 1.02 & 0.96 \\
$\mathrm{CO}_{2}(-4.4)$ & 1.05 & 1.03 & 0.98 \\
$\mathrm{C}_{6} \mathrm{~F}_{6}(17.2)$ & 1.23 & 1.03 & 1.07 \\
$\mathrm{c}_{-} \mathrm{C}_{6} \mathrm{H}_{12}(13.2)$ & 1.27 & 1.14 & 1.18 \\
$\mathrm{~N}_{2}(-1.4)$ & 0.79 & 0.80 & 0.78 \\
$\mathrm{C}_{6} \mathrm{H}_{6}(-5.6)$ & 1.05 & 1.07 & 1.04 \\
$\mathrm{CS}_{2}(6.1)$ & 1.06 & 1.01 & 1.03 \\
$\mathrm{CO}(0.4)$ & 1.01 & 1.01 & 1.01
\end{tabular}

( $\left.{ }^{a}\right)$ The numbers in parentheses are the values of the static quadrupole moments in $10^{-26}$ e.s.u. $\mathrm{cm}^{2}$.

$\left({ }^{b}\right)$ Results from Ref. [3]. In this work the static values of the quadrupole moment were used although the AQO model uses the ion-quadrupole interaction of Eq. (2).

averaged ion-quadrupole interaction depending on the square of the quadrupole moment must be similar for both $q Q>0$ and $q Q<0$ cases. The general agreement observed in tables I and II where the present method as well the statistical thermodynamic approach are applied for both $q Q>0$ and $q Q<0$ cases supports this view although of course detailed calculations must be carried out to elucidate this subject.

An interesting point that should be discussed are the high expt/theoretical values for the reactions of $\mathrm{OH}^{-}$and $\mathrm{CH}_{3} \mathrm{O}^{-}$with $\mathrm{C}_{2} \mathrm{H}_{2}$. The rate constants predicted by the theory are much too low compared to the experimental values. This discrepancy must rather reflect the neglect of high order contributions to the ion-molecule interaction potential such as the $r^{-6}$ term probably very significant in negative ion-molecule collisions, because negative ion polarizabilities could be very large, particularly in excited states [12].

We conclude that the present theory gives a good qualitative description of ion-quadrupolar molecule thermal reactions and leads to a simple equation which in view of all the approximations involved provides a good estimate of the reaction coefficient.

\section{References}

[1] Hirschfelder, J. O., Curtiss, C. F. and Bird, R. B., Molecular Theory of Gases and Liquids (Wiley, New York) 1964, p. 840.

[2] Dugan Jr., J. V. and Palmer, R. W., Chem. Phys. Lett. 13 (1972) 144.

[3] Su, T. and Bowers, M. T., Int. J. Mass Spectrom. Ion Phys. 17 (1975) 309.

[4] SU, T. and Bowers, M. T., Classical ion-molecule collision theory, in Gas Phase Ion Chemistry, ed. M. T. Bowers (Academic, New York) 1979.

[5] Celli, F., Weddle, G. and Ridge, D. P., J. Chem. Phys. 73 (1980) 801.

[6] Takayanagi, K., J. Phys. Soc. Jpn 51 (1982) 3337.

[7] BATES, D. R., Chem. Phys. Lett. 82 (1981) 396.

[8] Bates, D. R., Chem. Phys. Lett. 97 (1983) 19.

[9] Sakimoto, K., Chem. Phys. 85 (1984) 273.

[10] Abramowitz, M. and Stegun, I. A., Handbook of Mathematical Functions (Dover, New York) 1965.

[11] Mackay, G. I., Tanaka, K. and Bohme, D. K., Int. J. Mass Spectrom. Ion Phys. 24 (1977) 125.

[12] Bowers, M. T. and Su, T. in P. Ausloos ed. Interactions Between Ions and Molecules (Plenum, New York) 1975, p. 182. 